\title{
Phase transitions in diluted negative-weight percolation models
}

\author{
L. Apolo ${ }^{1,2}$ * O. Melchert ${ }^{2}$ 丹 and A. K. Hartmann 2 团 \\ 1 City College of the City University of New York, New York, New York 10031, USA \\ 2 Institut für Physik, Universität Oldenburg, Carl-von-Ossietzky Strasse, 26111 Oldenburg, Germany
}

(Dated: November 9, 2018)

\begin{abstract}
We investigate the geometric properties of loops on two-dimensional lattice graphs, where edge weights are drawn from a distribution that allows for positive and negative weights. We are interested in the appearance of spanning loops of total negative weight. The resulting percolation problem is fundamentally different from conventional percolation, as we have seen in a previous study of this model for the undiluted case.

Here, we investigate how the percolation transition is affected by additional dilution. We consider two types of dilution: either a certain fraction of edges exhibit zero weight, or a fraction of edges is even absent. We study these systems numerically using exact combinatorial optimization techniques based on suitable transformations of the graphs and applying matching algorithms. We perform a finite-size scaling analysis to obtain the phase diagram and determine the critical properties of the phase boundary. We find that the first type of dilution does not change the universality class compared to the undiluted case whereas the second type of dilution leads to a change of the universality class.
\end{abstract}

PACS numbers: 64.60.ah, 75.40.Mg, 02.60.Pn, 68.35.Rh

\section{INTRODUCTION}

Percolation is one of the most fundamental problems in statistical mechanics [4, 5]. Its ideas apply to physical disciplines as different as solid-state physics and cosmology and it has the ability to describe many phase transitions in a completely geometric and probabilistic context. The pivotal question in percolation theory is that of connectivity. Consider for example random-bond percolation where one studies a lattice that is randomly filled with a certain fraction of edges. Clusters of connected sites are then analyzed regarding their geometric properties. Depending on the fraction of edges on the lattice, the geometric properties of the clusters change, leading from a phase with rather small and disconnected clusters to a phase where there is basically one large cluster dominating the lattice. Therein, the appearance of an infinite, i.e. percolating, cluster is described by a second order phase transition. In the past decades, a large number of percolation problems have been investigated in various contexts through numerical simulations. Among those are problems where the objects of interest are string-like, rather than clusters as in the standard case explained above. The geometric properties of stringlike objects have been studied and quantified in diverse contexts [2, 3, 7, 9, 11, 14, 17, 19]. Recently [16] we have investigated negative weight percolation (NWP), a problem in similar vein but with subtle differences compared to other string-like percolation problems. In NWP, we consider a regular lattice with periodic boundary conditions where all adjacent sites are joined by undirected

\footnotetext{
*Electronic address: lapolo00@ccny.cuny.edu

${ }^{\dagger}$ Electronic address: melchert@theorie.physik.uni-oldenburg.de

${ }^{\ddagger}$ Electronic address: alexander.hartmann@uni-oldenburg.de
}

edges. Weights are assigned to the edges, representing quenched random variables drawn from a distribution that allows for edge weights of either sign. The details of the weight distribution is therein controlled by a disorder parameter. For a given realization of the disorder, we then ask for a configuration of loops, i.e. closed polygons on the lattice, such that the sum of the edge weights that make up the loops is minimal. The problem of finding these loops can be cast into a minimum-weight-path (MWP) problem as will be explained below in more detail. This MWP is also of relevance for the problem of finding domain-wall excitations in 2d Ising spin glasses 15]. One feature of this particular MWP problem is that there is no modularity, meaning that a path obeying the optimization constraint can have subpaths that do not meet the optimization criterion. We further impose the constraint that the loops are not allowed to intersect, as a result there is no definition of clusters in NWP.

Previously we investigated NWP on two-dimensional lattices. We found a critical value of the disorder parameter above which percolating loops emerge in the limit of large system sizes. We further determined a whole set of critical exponents that characterize the underlying disorder-induced and geometric phase transition. These exponents where clearly different from those describing other percolation transitions. Moreover, the critical exponents where found to be universal in $2 d$, meaning that they do not depend on the details of the disorder distribution or the lattice geometry.

Albeit there are essential differences between NWP and usual bond percolation described above, the features of the latter are important if we ask for the effect of dilution on the characteristics of NWP. Here, we investigate the effect of dilution on the critical properties of NWP. Therefore we use observables from percolation theory and a finite-size-scaling (FSS) analysis to probe critical lines 
(a)

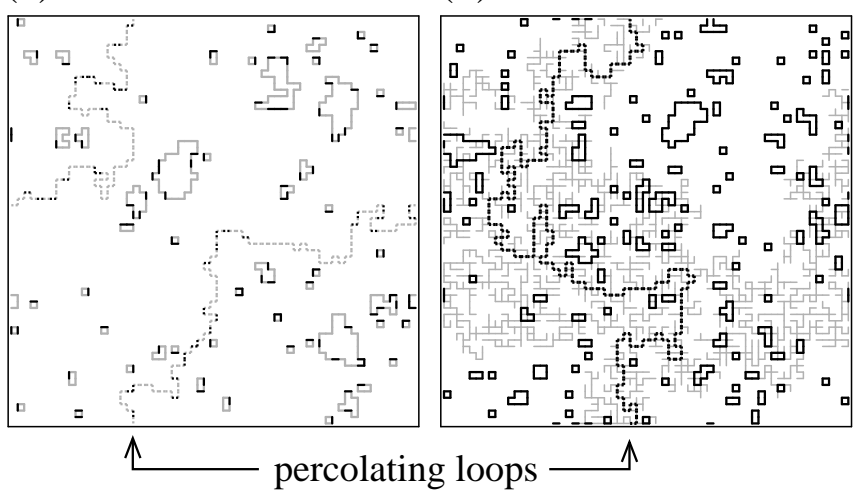

FIG. 1: Samples of $L=64$ loop configurations for the two different types of disorder investigated. Percolating loops appear as dashed lines and are marked by arrows. (a) $\rho=0.1$, $p_{\mathrm{I}}=0.3940$, where $p_{\mathrm{I}}$ is the fraction of edges with weight equal to zero. A loop can include those edges without increasing its configurational energy. Edges with zero weight that are included in loops are colored grey. (b) $\rho=1.0, p_{\text {II }}=0.4998$, where $p_{\text {II }}$ is the fraction of missing edges on the lattice. Those edges that comprise the cluster of connected edges on which the percolating loop is located appear as thin grey lines.

in the disorder-dilution plane for two different types of dilution described below. Further, we check whether the critical exponents change along the critical lines. We therefore analyze the critical exponent $\nu$ that describes the divergence of a typical length scale in NWP as we approach the critical point. For one distinguished point in the disorder-dilution plane, a more complete analysis involving more critical exponents is given as well.

The paper is organized as follows. In section [I we introduce the model in more detail and we outline the algorithm we used to obtain the loop configurations. In section [II], we list the results of our numerical simulations followed by a summary and conclusions in section IV.

\section{MODEL AND ALGORITHM}

In the framework of this paper, we consider $2 d$ square lattices $G=(\mathcal{V}, \mathcal{E})$ with side length $L$ and fully periodic boundary conditions (BCs), i.e. $N=L \times L$ sites $i \in \mathcal{V}$ and a maximal number of $2 N$ (in case of no dilution) edges $\{i, j\} \in \mathcal{E}$ joining adjacent sites $i, j \in \mathcal{V}$. We further assign a weight/cost $\omega_{i j}$ to each edge contained in $\mathcal{E}$, representing quenched random variables that introduce disorder to the lattice. One realization of the undiluted disorder therein consists of a fraction $\rho$ of edge weights drawn from a Gaussian distribution with zero mean and unit width, together with edges with unit weight (fraction $1-\rho$ ). This weight distribution explicitly allows for loops $\mathcal{L}$, i.e. closed polygons on the lattice, with a negative total weight $\omega_{\mathcal{L}}=\sum_{\{i, j\} \in \mathcal{L}} \omega_{i j}$. For any nonzero value of the disorder parameter $\rho$, a sufficiently large lattice will exhibit at least small loops with a weight smaller than zero. If the disorder parameter is sufficiently large, even large loops with negative weight will appear, which span the system. Hence, the system is percolating in this case. Here, as an additional source of disorder, we consider two different types of dilution, affecting the lattice edges:

- Type I: The disorder is characterized by a fraction $p_{\text {I }}$ of edge weights $\omega_{i j}=0$. This does not mean that the respective bonds are absent in terms of bond percolation. Instead, a loop can include these edges without an increase of the configurational energy, see figure 1(a);

- Type II: The lattice is diluted by a fraction $p_{\text {II }}$ of absent bonds. Depending on the value of $p_{\text {II }}$, this induces a usual random bond percolation process that will affect the negative weight percolation (NWP) of loops, see figure 1(b).

Given $G$ together with a realization of the disorder, we determine a set $\mathcal{C}$ of loops such that the configurational energy, defined as the sum of all the loop-weights $E=\sum_{\mathcal{L} \in \mathcal{C}} \omega_{\mathcal{L}}$, is minimized. As further optimization constraint, the loops are not allowed to intersect and generally, the weight of an individual loop is smaller than zero. Note that $\mathcal{C}$ may also be empty. Clearly, the configurational energy $E$ is the quantity subject to optimization and the result of the optimization procedure is a set of loops $\mathcal{C}$, obtained using an appropriate transformation of the original graph as detailed in [12]. So as to identify the edges that constitute the loops for a particular instance of the disorder, we need to obtain a minimum-weighted perfect matching (MWPM) [13, 20] on the transformed graph.

Here, we give a brief description of the algorithmic procedure that yields a minimum weight set of loops for a given realization of the disorder. Figure 2 illustrates the 3 basic steps, detailed below:

(1) each edge, joining adjacent sites on the original graph $G$, is replaced by a path of 3 edges. Therefore, 2 "additional" sites have to be introduced for each edge in $\mathcal{E}$. Therein, one of the two edges connecting an additional site to an original site gets the same weight as the corresponding edge in $G$. The remaining two edges get zero weight. The original sites $i \in \mathcal{V}$ are then "duplicated", i.e. $i \rightarrow i_{1}, i_{2}$, along with all their incident edges and the corresponding weights. For each of these pairs of duplicated sites, one additional edge $\left\{i_{1}, i_{2}\right\}$ with zero weight is added that connects the two sites $i_{1}$ and $i_{2}$. The resulting auxiliary graph $G_{\mathrm{A}}=\left(\mathcal{V}_{\mathrm{A}}, \mathcal{E}_{\mathrm{A}}\right)$ is shown in figure 2(b), where additional sites appear as squares and duplicated sites as circles. Figure2(b) also illustrates the weight assignment on the transformed graph $G_{\mathrm{A}}$. Note that while the original graph (figure 2(a)) is symmetric, the transformed graph (figure 2(b)) is not. This is due to the details of the mapping procedure and the particular weight assignment we have chosen. A more extensive description of the mapping can be found in [15].

(2) a MWPM on the auxiliary graph is determined via exact combinatorial optimization algorithms [1]. A 
(a)

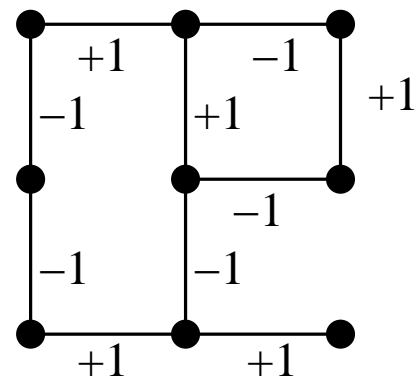

(c)

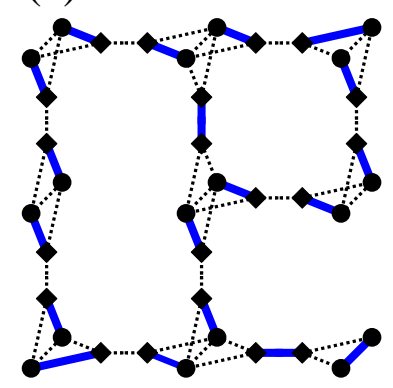

(b)

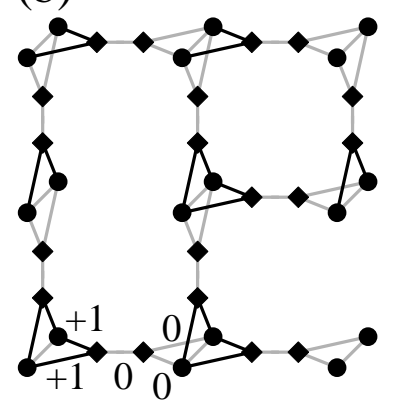

(d)

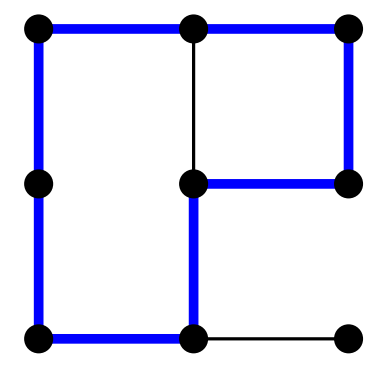

FIG. 2: Illustration of the algorithmic procedure. (a) original lattice $G$ with edge weights, (b) auxiliary graph $G_{\mathrm{A}}$ with proper weight assignment: black edges carry the same weight as the respective edge in the original graph and grey edges carry zero weight. (c) minimum-weight perfect matching $M$ : bold edges are matched and dashed edges are unmatched. (d) loop configuration (bold edges) that corresponds to the MWPM depicted in (c).

MWPM is a minimum-weighted subset $M$ of $\mathcal{E}_{\mathrm{A}}$, such that each site contained in $\mathcal{V}_{\mathrm{A}}$ is met by precisely one edge in $M$. This is illustrated in figure 2(c), where the solid edges represent $M$ for the given weight assignment. The dashed edges are not matched. Due to construction, the auxiliary graph consists of an even number of sites and since there are no isolated sites, it is guaranteed that a perfect matching exists. Even if theres no edge on $G$, i.e. $p_{\mathrm{II}}=1$ in terms of the dilution introduced above. In that extreme case, $G_{\mathrm{A}}$ consists solely of the "duplicated" sites that are joined pairwise by zero-weighted edges. A perfect matching trivially exists and the corresponding MWPM has weight zero.

(3) finally it is possible to find a relation between the matched edges $M$ on $G_{\mathrm{A}}$ and a configuration of negativeweighted loops $\mathcal{C}$ on $G$ by tracing the steps of the transformation (1) back. In this regard note that each edge contained in $M$ that connects an additional site (square) to a duplicated site (circle) corresponds to an edge on $G$ that is part of a loop, see figure 2(d). More precisely, there are always two such edges in $M$ that correspond to one loop segment on $G$. All the edges in $M$ that connect like sites (i.e. duplicated-duplicated, or additionaladditional) carry zero weight and do not contribute to a loop on $G$. Once the set $\mathcal{C}$ of loops is found, a depth-first search [12, 13] can be used to explore $\mathcal{C}$ and to determine

the geometric properties of the individual loops. For the weight assignment in figure 2(a), there is only one loop with weight $\omega_{\mathcal{L}}=-2$ and length $\ell=8$.

Note that the result of the calculation is a collection $\mathcal{C}$ of loops such that the total loop weight, and consequently the configurational energy $E$, is minimized. Hence, one obtains a global collective optimum of the system. Obviously, all loops that contribute to $\mathcal{C}$ possess a negative weight. Regarding the weight assignment in step (1) there are different possibilities that all result in equivalent sets of matched edges on the transformed lattice, corresponding to the minimum-weight collection of loops on the original lattice. Some of these weight assignments lead to a more symmetric transformed graph, see e.g. [12]. However, this is only a technical issue that does not affect the resulting loop configuration. Albeit the transformed graph is not symmetric, the resulting graph (figure 2(d)) is again symmetric.

In the following we will use the procedure outlined above to investigate the effect of dilution on the critical properties of NWP.

\section{RESULTS}

So as to clarify the effect of dilution on the critical properties of NWP, we first need to locate the critical lines in the $\rho-p_{\mathrm{I} / \mathrm{II}}$-planes that separate the percolating from the nonpercolating domains. Therefore, we use observables from percolation theory and a finitesize-scaling analysis. Besides the weight $\omega_{\mathcal{L}}$ and length $\ell=\sum_{\{i, j\} \in \mathcal{L}} 1$ of an individual loop, we determine its linear extensions by projecting it onto the perpendicular lattice axes. The larger of the two is called the spanning length of the loop and the smaller one is called its roughness. Consequently, a loop is called percolating if its spanning length is equal to the system size $L$. This is a binary decision for each disorder instance that is further used to obtain the percolation probability $P_{L}\left(\rho, p_{\mathrm{I} / \mathrm{II}}\right)$ for a lattice of a certain size at a given value of the disorder and dilution parameters $\rho$ and $p=p_{\mathrm{I} / \mathrm{II}}$, respectively. Note that system spanning loops are a direct consequence of the periodic boundary conditions we employ. For completeness, we note that we used the number 1 to signify a sample containing a percolating loop and 0 otherwise. In practice, we fix one of the parameters, say $\rho$, and tune the value of $p$ so as to determine the critical point $p_{c}$ beyond which percolating loops emerge in the limit of large system sizes at that particular value of $\rho$. For clarity, $P_{L}(\rho, p)$ at fixed $\rho$ is simply denoted as $P_{L}(p)$. Referring to percolation theory [5], $P_{L}(p)$ is expected to scale as $P_{L}(p) \sim f_{1}\left[\left(p-p_{c}\right) L^{1 / \nu}\right]$, where $\nu$ denotes the critical exponent that describes the divergence of the typical length scale as the critical point is approached. Since we consider string-like objects, it is possible to find more than just one percolating loop per disorder instance. In a preceding work [16] we found that the average number of percolating loops per sample satisfies the scaling relation 
(a)

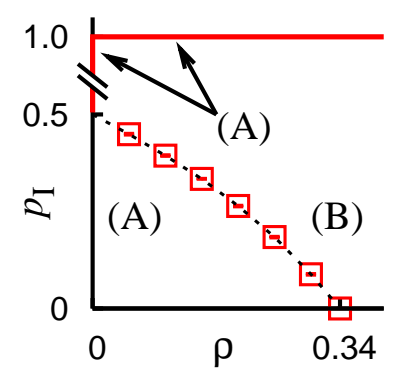

(b)

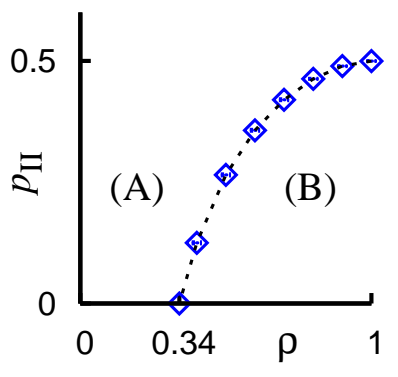

FIG. 3: Critical lines in the disorder-dilution planes. Phase diagram for type I and type II dilution, (a) and (b) respectively. (A) signifies a domain in the disorder-dilution plane without percolating loops and (B) labels a domain where percolating loops are present.

$\langle N\rangle \sim f_{2}\left[\left(p-p_{c}\right) L^{1 / \nu}\right]$ governed by the same values for $p_{c}$ and $\nu$ as the percolation probability. Therein $\langle N\rangle$ was found to exceed a value of 1 above the critical point, similar to what was found earlier for a different loop percolation model subject to optimization constraints [17]. This is in contrast to usual random-bond percolation, where the objects of interest are cluster of connected sites on the lattice and where there is a unique percolating cluster above the percolation threshold.

\section{A. Type I dilution}

As described above, we allow for a fraction $p_{\text {I }}$ of edges that carry zero weight. A loop can include those edges without increasing its weight or the configurational energy. As a consequence, for values of $p_{\text {I }}>0$ percolating loops can emerge even if the disorder parameter $\rho$ has

TABLE I: Critical points in the disorder-dilution plane for type I dilution. From left to right: numerical value of the disorder $(\rho)$ parameter and the dilution $\left(p_{\mathrm{I}}\right)$ along the critical line (the values without errorbars where kept fixed) and critical exponent $\nu$ that describes the divergence of the correlation length. $S$ measures the quality of the data collapse as the mean-square distance of the data sets to the master curve in units of the standard error.

\begin{tabular}{llll}
\hline \hline$\rho$ & $p_{\text {I }}$ & $\nu$ & $S$ \\
\hline 0.05 & $0.449(2)$ & $1.49(13)$ & 1.19 \\
0.10 & $0.394(2)$ & $1.52(14)$ & 1.19 \\
0.15 & $0.334(1)$ & $1.52(2)$ & 1.43 \\
0.20 & $0.264(2)$ & $1.52(9)$ & 1.00 \\
$0.200(2)$ & 0.264 & $1.45(12)$ & 0.91 \\
0.25 & $0.184(2)$ & $1.52(11)$ & 1.21 \\
$0.249(2)$ & 0.184 & $1.51(14)$ & 0.91 \\
0.30 & $0.088(2)$ & $1.51(7)$ & 0.72 \\
$0.340(1)$ & 0.000 & $1.49(7)$ & 0.91 \\
\hline \hline
\end{tabular}
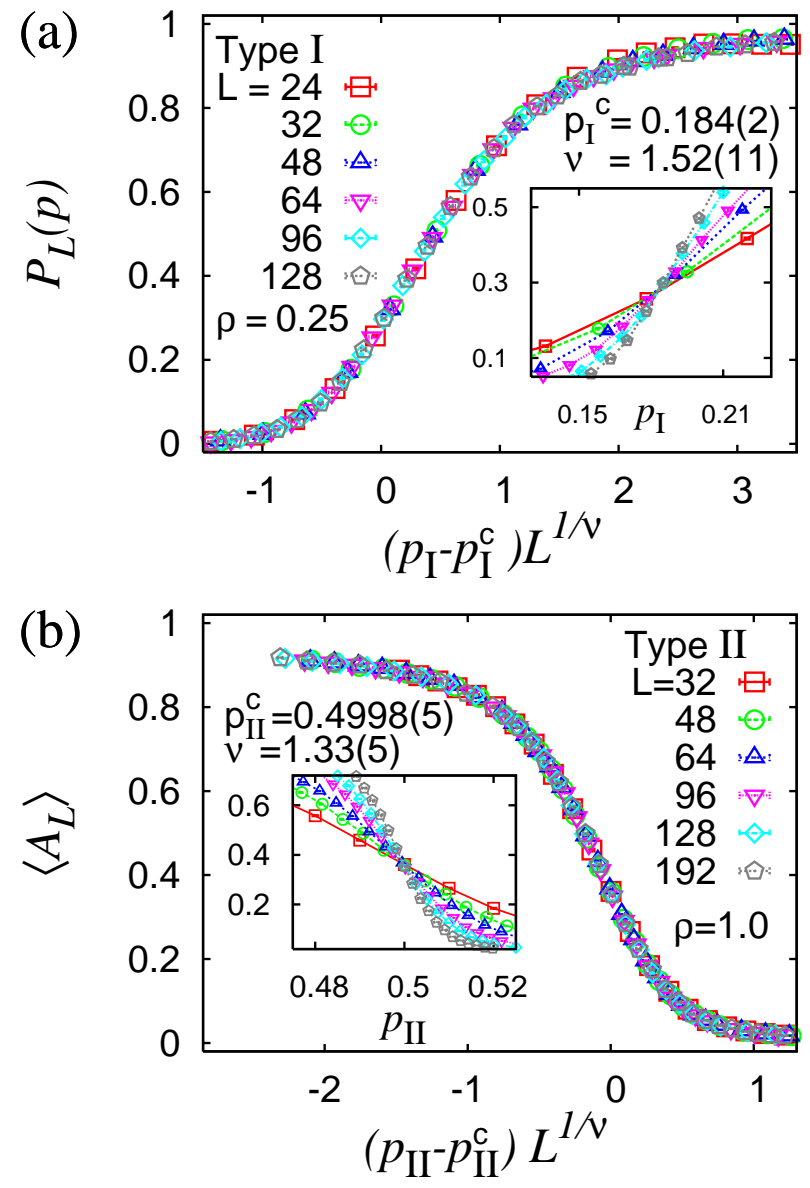

FIG. 4: Scaling analysis to probe points along the critical lines in the disorder-dilution planes. (a) For a disorder parameter fixed to $\rho=0.25$, the scaling analysis of the percolation probability $P_{L}$ yields a critical value $p_{\mathrm{I}}^{\mathrm{c}}=0.184(2)$ for the dilution above which percolating loops appear in the system. It further yields a critical exponent $\nu=1.52(11)$. (b) For a disorder parameter fixed to $\rho=1.0$, the scaling analysis of the normalized average box size $A_{L}$ yields a critical value $p_{\mathrm{II}}^{\mathrm{c}}=0.4998(5)$ for the dilution parameter and a critical exponent $\nu=1.33(5)$.

a value smaller than its critical value along the $p_{\mathrm{I}}=0$ axis, i.e. $\rho=0.34$. In particular for $p_{\mathrm{I}}>0.5$, there is a system spanning cluster of edges with zero weight and for $L \rightarrow \infty$ an arbitrarily small value of $\rho$ will suffice to generate percolating loops on the lattice.

First, we probed the critical line that separates the nonpercolating (small $p_{\mathrm{I}}$ at fixed $\rho$ ) and the percolating domains in the disorder-dilution plane. For this purpose, we performed simulations for systems of size $L=24 \ldots 128$, the number of samples ranging from 32000 for $L=24$ to 12800 for the largest systems. Within the scaling analysis, we fixed the disorder parameter $\rho$ at values listed in table $\square$ and varied the dilution $p_{\mathrm{I}}$ so as to determine the critical point $p_{\mathrm{I}}^{\mathrm{c}}$ at which percolating loops emerge. As an indicator for the transition, we therefore study the finite-size-percolation probability $P_{L}\left(p_{\mathrm{I}}\right)$. The 
resulting phase diagram is depicted in figure 3(a) and a scaling analysis for one of the points along the critical line is shown in figure 4(a). A data collapse involving data sets for different system sizes $L$ can be used to estimate $p_{\mathrm{I}}^{\mathrm{c}}$ and $\nu$. The quality of the data collapse is therein measured by the mean square distance $S$ of the data sets to the master curve in units of the standard error [10], see table [1. For the example illustrated in figure 4(a), where the disorder parameter was fixed to $\rho=0.25$, the scaling analysis resulted in a value $p_{c}=0.184(2)$ for the dilution above which percolating loops are present in the system. It further yields a value $\nu=1.52(11)$ for the critical exponent that describes the divergence of the correlation length. For consistency, we checked some of the points by performing simulations along both axis: in addition to the data obtained at fixed $\rho$ we performed simulations at fixed $p_{\mathrm{I}}$, probing the critical point along the $\rho$-axis. For example, probing along the $p_{\mathrm{I}}$-axis at $\rho=0.2$ we found $p_{\mathrm{I}}^{\mathrm{c}}=0.264(2)$, while probing along the $\rho$-axis at $p_{\mathrm{I}}=0.264$ we obtained $\rho=0.200(2)$ in complete agreement. Albeit the sample numbers in the present study are somewhat smaller than in [16] that led to the estimate of $\nu$ at $p_{\mathrm{I}}=0$, the numerical values we obtain for $\nu$ along the critical line are consistent with the value of $\nu=1.49(7)$ reported there. This leads us to conclude that type I dilution does not change the universality class of NWP. Note that it is not possible to find negative weighted loops along the $p_{\mathrm{I}}=1$ and $\rho=0$ axes since at least one edge with a negative edge weight is need in order to form a loop.

\section{B. Type II dilution}

As detailed above, we allow for a fraction $p_{\text {II }}$ of absent edges on the lattice. In terms of random-bond percolation, one can expect that the lattice decomposes into independent clusters of connected sites for $p_{\text {II }}>1 / 2$. Therefore its very unlikely to find negative weighted loops

TABLE II: Critical points in the disorder-dilution plane for type II dilution. From left to right: numerical value of the disorder $(\rho)$ and dilution $\left(p_{\text {II }}\right)$ parameter along the critical line (the values without errorbars where kept fixed) and critical exponent $\nu$ that describes the divergence of the correlation length. $S$ measures the quality of the data collapse as the mean square distance of the data sets to the master curve in units of the standard error.

\begin{tabular}{llll}
\hline \hline$\rho$ & $p_{\text {II }}$ & $\nu$ & $S$ \\
\hline $0.340(1)$ & 0.0 & $1.49(7)$ & 0.91 \\
0.4 & $0.125(2)$ & $1.49(9)$ & 0.97 \\
0.5 & $0.265(2)$ & $1.49(15)$ & 1.02 \\
0.6 & $0.357(2)$ & $1.49(11)$ & 1.03 \\
0.7 & $0.420(2)$ & $1.47(11)$ & 1.02 \\
0.8 & $0.463(1)$ & $1.47(11)$ & 0.93 \\
0.9 & $0.4893(9)$ & $1.41(8)$ & 1.20 \\
1.0 & $0.4998(5)$ & $1.33(5)$ & 0.98 \\
\hline \hline
\end{tabular}

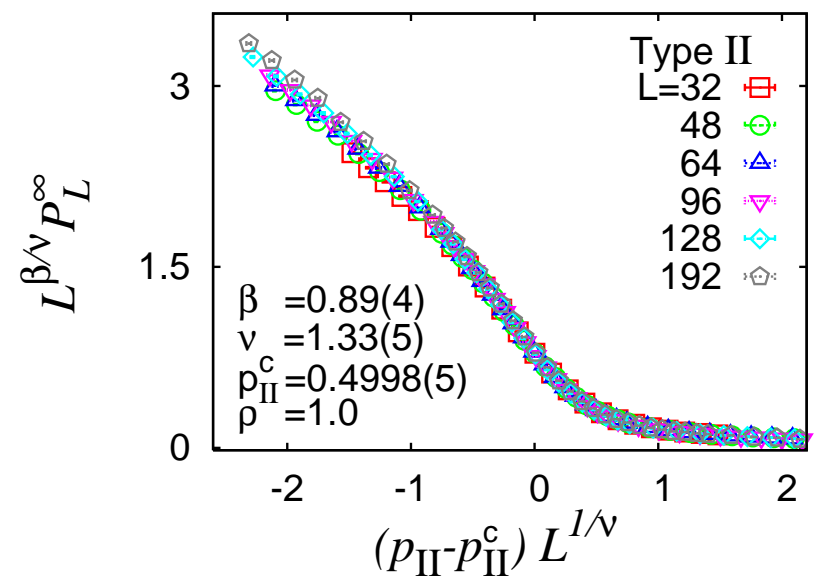

FIG. 5: FSS of the average normalized loop length $P_{L}^{\infty}$ that leads to the estimate of $\beta$ listed in table III Here, the data collapse is best near the critical point while there are corrections to scaling off criticality.
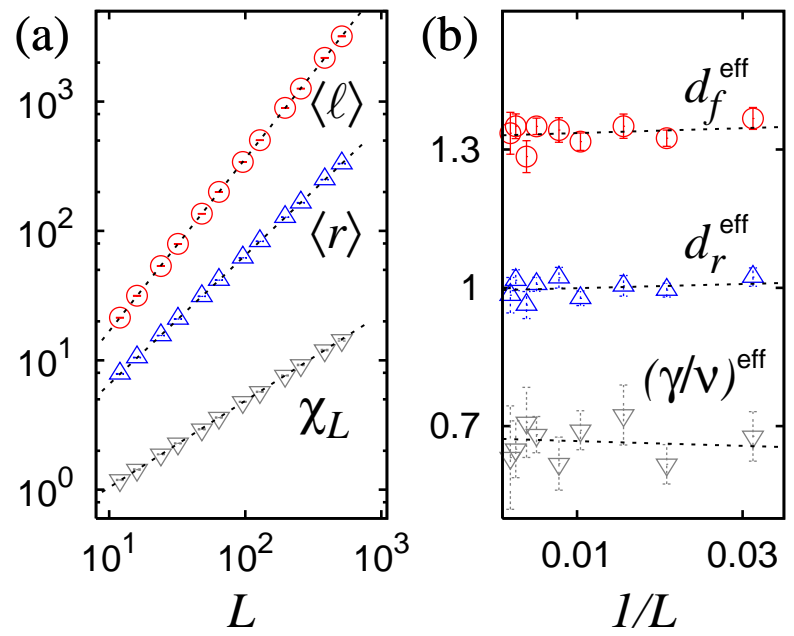

FIG. 6: Analysis of the fractal dimension $d_{f}$, the roughness exponent $d_{r}$ and the exponent $\gamma / \nu$ that relates to the fluctuations of the loop lengths at $\rho=1.0$ and $p_{\text {II }}=0.4998(5)$. (a) Estimation of the above exponents by means of a pure fit to the power law data. (b) Estimation of the asymptotic exponents by extrapolation using the effective (local) exponents of the data points shown in (a).

with a spanning length equal to $L$ for values of the dilution that exceed $p_{\text {II }}=1 / 2$. Proceeding as above, we fixed the value of the disorder parameter to the values listed in table $\Pi$ and tuned the value of the dilution parameter so as to probe the corresponding critical points $p_{\mathrm{II}}^{\mathrm{c}}$, above which percolating loops cease to exist (large $p_{\text {II }}$ at fixed $\rho$ ). We performed simulations for systems of size $L=32 \ldots 128$. The number of samples ranged from 19200 for $L=32$ to 12800 for the largest systems. We performed in each case a FSS analysis. Only at $\rho=1.0$ we carried out further simulations at $L=192$ 
(9600 samples) so as to decrease finite-size effects furthermore and to establish a more reliable scaling analysis to settle a value for the critical exponent $\nu$. Figure 4(b) illustrates a scaling analysis for fixed $\rho=1.0$, corresponding to the rightmost data point in figure 3(b). Instead of the percolation probability shown for the type I dilution in figure $4(\mathrm{a})$, we here show the average area $\left\langle A_{L}\right\rangle \equiv\left\langle\ell_{x} \times \ell_{y}\right\rangle / L^{2}$ of the smallest box that fits the largest loop on the lattice normalized by the system size. In the same manner as the percolation probability, this quantity approaches a step function in the limit of large system sizes and its finite size scaling behavior follows the analogous form $\left\langle A_{L}\right\rangle \sim f_{3}\left[\left(p_{\text {II }}-p_{\text {II }}^{\text {c }}\right) L^{1 / \nu}\right]$. For fixed $\rho=1.0$ the FSS of the observable $A_{L}$ yields a critical value $p_{\mathrm{II}}^{\mathrm{c}}=0.4998(5)$ of the dilution parameter and the critical exponent $\nu=1.33(5)$ with a quality $S=1.25$ of the data collapse. These findings are in complete agreement with the corresponding values listed in table $\amalg$ that where obtained by means of the percolation probability. It is interesting to note that the exponent $\nu$ steadily decreases from the value $\nu=1.49(7)$ found for the undiluted model at $\rho=0.340(1)$ to the value $\nu=1.33(5)$ at $\rho=1.0$ and $p_{\text {II }}=0.4998(5)$, see table III. The latter two values are also characteristic for random bond percolation in $2 \mathrm{~d}$, that is the accompanying process for NWP subject to the type of dilution under investigation. The change of the exponent $\nu$ indicates the introduction of type II dilution changes the universality class of NWP. In particular, since the value of $\nu$ is compatible with the standard percolation exponent, we ask the question whether the NWP is here equivalent to standard percolation, although we look for loops of negative weight instead for percolating clusters. In the following we present a more detailed analysis of the critical point at $\rho=1.0$ and $p_{\text {II }}=0.4998(5)$, which shows that indeed the NWP behavior at $\rho=1.0$ is not equivalent to standard percolation.

\section{Detailed analysis at $\rho=1.0$ and $p_{\mathrm{II}}^{\mathrm{c}}=0.4998(5)$}

Above we found the critical point $p_{\mathrm{II}}^{\mathrm{c}}$ and the exponent $\nu$ from a FSS analysis of the average normalized box size $A_{L}$. Further, the average normalized loop length $P_{L}^{\infty} \equiv$ $\langle\ell\rangle / L^{2}$ can be used to obtain a further critical exponent, namely the percolation strength $\beta$, by use of the scaling assumption $P_{L}^{\infty} \sim L^{-\beta / \nu} f_{4}\left[\left(p_{\mathrm{II}}-p_{\mathrm{II}}^{\mathrm{c}}\right) L^{1 / \nu}\right]$ as suggested by the corresponding observables known from percolation theory. From the FSS of $P_{L}^{\infty}$ we obtained the critical exponent $\beta=0.89(6)$ with a quality $S=0.97$ of the scaling assumption, see figure 5 table [II. In the formula above, the value of $\ell$ refers to the length of the largest loop found for a given realization of the disorder. At criticality this reduces to $P_{L}^{\infty} \sim L^{-\beta / \nu}$.

Next, we consider the fractal (scaling) dimension of the loops defined via $\langle\ell\rangle \sim L^{d_{f}}$. The value of $d_{f}$ is bounded by the intrinsic dimension of a line and the topological dimension of the environment to $1 \leq d_{f} \leq 2$. From scal-

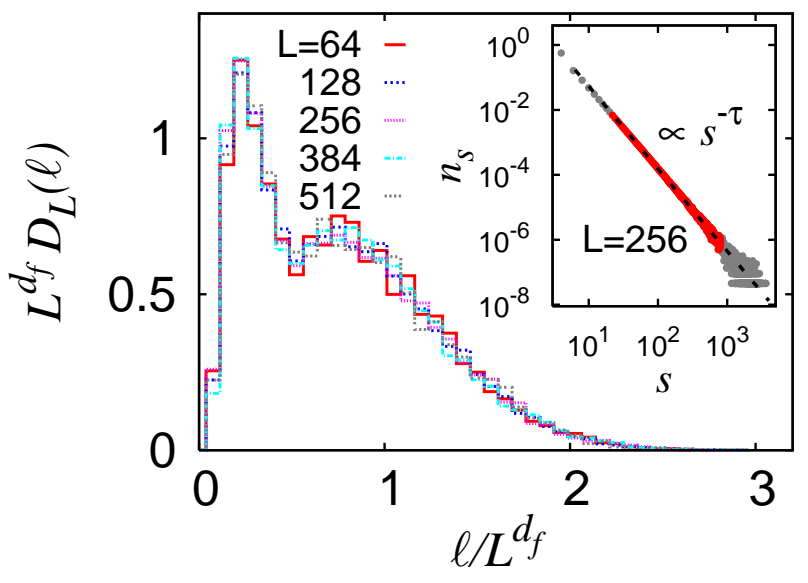

FIG. 7: Detailed analysis at $\rho=1.0$ and $p_{\text {II }}$. Rescaled distribution $D_{L}(\ell)$ of the length $\ell$ of the largest loop found for each realization of the disorder. The inset shows the distribution $n_{s}$ of all loop-lengths $s$, excluding the spanning loops, at the critical point. Grey data points were omitted from the fit that led to the estimate of $\tau=2.54(5)$.

ing theory, we obtain the relation $d_{f}=2-\beta / \nu$. This scaling relation may further be used to check for consistency as we employ two different methods to estimate critical exponents, i.e. a data collapse involving data at different values of $p_{\text {II }}$ and an analysis of the algebraic scaling of different observables right at the critical point. A simple fit to the power law data, shown in figure 6(a), yields the exponent $d_{f}=1.333(2)$ with quality $Q=0.51$ [8]. This is compatible with the scaling relation within error bars, given the values deter minded above for $\beta$ and $\nu$. Interestingly, the value of $d_{f}$ found here coincides with the fractal dimension of self avoiding walks on a regular lattice $\left(d_{f}^{\mathrm{SAW}}=4 / 3\right)$ and of exterior perimeters measured for $2 d$ percolation clusters at the percolation threshold [6]. Moreover, it compares well with the value $d_{f}^{\mathrm{BB}}=1.34(2)$ that describes the scaling of the average backbone perimeter with system size, measured for random percolation at the percolation threshold [18]. This suggests, in analogy with the conclusions of [18], that negative weighted loops at $\left(\rho, p_{\text {II }}\right)=(1.0,0.4998)$ belong to the same universality class as self-avoiding walks. The roughness of the loops is expected to display the critical scaling $\langle r\rangle \sim L^{d_{r}}$ with a roughness exponent $d_{r}=1$. The fit to the power law data, also shown in figure 6(a), yields indeed the exponent $d_{r}=0.998(2)$ with quality $Q=0.33$, highlighting the self-similar nature of the loops.

The susceptibility like quantity $\chi_{L}=\left(\left\langle\ell^{2}\right\rangle-\langle\ell\rangle^{2}\right) / L^{2}$, that measures the mean square fluctuation of the loop length, exhibits the critical scaling $\chi_{L} \sim L^{\gamma / \nu}$ that allows to determine the critical exponent $\gamma=0.89(4)$ with a quality $Q=0.56$ of the fit, see again figure 6(a). It is connected to the other exponents via the scaling relation $\gamma+2 \beta=2 \nu$, which is fulfilled within error bars.

Note that an analysis of the effective (local) exponents of the data belonging to $\langle\ell\rangle,\langle r\rangle$ and $\chi_{L}$ yields the asymp- 
TABLE III: Critical points and exponents. From left to right: Type (RBP: random bond percolation, NWP: negative weight percolation ), dilution $\left(p_{\mathrm{II}}\right)$ and disorder $(\rho)$ parameters (note that there is no entry for RBP since the disorder parameter $\rho$ introduced here has no analog in usual random percolation), critical exponent of the correlation length $\nu$, percolation strength $\beta$, exponent $\gamma$, Fisher exponent $\tau$ and fractal dimension $d_{f}$ at criticality.

\begin{tabular}{llllllll}
\hline \hline Type & $p_{\text {II }}$ & $\rho_{c}$ & $\nu$ & $\beta$ & $\gamma$ & \multicolumn{1}{l}{} \\
\hline RBP & 0.5 & - & $4 / 3$ & $5 / 36$ & $43 / 18$ & $187 / 91$ \\
NWP & 0.0 & $0.340(1)$ & $1.49(7)$ & $1.07(6)$ & $0.77(7)$ & $2.59(3)$ & $1.266(2)$ \\
NWP & $0.4998(5)$ & 1.0 & $1.33(5)$ & $0.89(4)$ & $0.89(6)$ & $2.54(5)$ & $1.333(2)$ \\
\hline \hline
\end{tabular}

totic scaling exponents $d_{f}=1.33(1), d_{r}=0.99(1)$ and $\gamma=0.89(6)$ compatible with the values found above but with somewhat larger errorbars, see figure 6(b).

As evident from figure 7 , the distributions $D_{L}(\ell)$ of the largest loops $\ell$ found for each realization of the disorder for different system sizes $L$ show a nice data collapse after a rescaling to the form $D_{L}(\ell)=L^{-d_{f}} f_{5}\left[\ell / L^{d_{f}}\right]$. This means that not only the average $\langle\ell\rangle$ but the full distribution scales with $d_{f}$, again highlighting the fractal nature of the loops. Therein, the peak close to $\ell / L^{d_{f}}=$ 0.25 stems from the non-spanning loops and the peak close to $\ell / L^{d_{f}}=0.75$ stems from those that truly span the lattice along at least one direction. At the critical point, we further expect the distribution $n_{s}$ of all loop lengths $s$, excluding loops with a spanning length $L$, to exhibit an algebraic scaling $n_{s} \sim s^{-\tau}$, where $\tau$ signifies the Fisher exponent. The exponent $\tau$ characterizes the complete ensemble of loops and it is related to the fractal exponent of the loops via $\tau-1=d / d_{f}$. Here, we found a value of $\tau=2.54(5)$, see inset of figure 7 in agreement with the latter scaling relation.

\section{CONCLUSIONS}

In summary, we performed numerical simulations in order to probe the effect of dilution on the critical properties of NWP on $2 d$ lattice graphs. In this context and by means of exact combinatorial optimization algorithms we have investigated two different types of dilution. One type affecting the distribution of weights that are associated with the edges, the other type having an impact on the topology of the underlying lattice. We used observables from percolation theory and a FSS analysis so as to probe critical points along the critical lines in the disorder-dilution plane that separate domains that allow/disallow system spanning, i.e. percolating, loops. We found that the first type of dilution does not change the universality class of NWP, i.e. the critical exponents are the same as for the undiluted model. The second type of dilution very well leads to a change of the universality class: As the disorder on the lattice increases, the critical exponent $\nu$ as well as the critical point of dilution approach the corresponding values known from usual random bond percolation, although the process, also due to its string-like nature, is clearly different from standard percolation, also visible through the values of the other critical exponents. A more detailed analysis of the critical exponents for one particular critical point in the disorder-dilution plane, i.e. $\left(\rho, p_{\mathrm{II}}\right)=(1.0,0.4998)$, verified that the exponents are connected by the usual scaling relations. Moreover, the results for the scaling behavior of the percolating loops suggest that negative weighted loops at that particular critical point belong to the same universality class as self-avoiding walks.

\section{Acknowledgments}

LA acknowledges a scholarship of the German academic exchange service DAAD within the "Research Internships in Science and Engineering" (RISE) program and the City College Fellowship program for further support. We further acknowledge financial support from the VolkswagenStiftung (Germany) within the program "Nachwuchsgruppen an Universitäten". The simulations were performed at the "Gesellschaft für Wissenschaftliche Datenverarbeitung" in Göttingen (Germany), and the GOLEM I cluster for scientific computing at the University of Oldenburg (Germany).
[1] For the calculation of minimum-weighted perfect matchings we use Cook and Rohes blossom4 extension to the Concorde library.

[2] Krinner A. Bittner E. and Janke W. Vortex-line percolation in the three-dimensional complex ginzburg-landau model. Phys. Rev. B, 72(9):094511, 2005.

[3] Antunes N. D. and Bettencourt L. M. A. The Length Distribution of Vortex Strings in $U(1)$ Equilibrium Scalar Field Theory. Phys. Rev. Lett., 81(15), 1998.
[4] Stauffer D. Scaling theory of percolation clusters. Phys. Rep., 54(1):1-45, 1979.

[5] Stauffer D. and Aharony A. Introduction to Percolation Theory. Taylor and Francis, 1994.

[6] Freund H. and Grassberger P. Percolation and selfavoiding random loops. J. Phys. A: Mathematical and General, 24(7):L383-L387, 1991.

[7] Hindmarsch H. and Strobl K. Statistical properties of strings. Nucl. Phys. B, 437:471, 1995. 
[8] Press W. H., Teukolsky S. A., Vetterling W. T., and Flannery B. P. Numerical Recipes in C, 1992. $Q$ is the probability that the value of $\chi^{2}$ is worse than in the current fit, under the assumption that the fitted function in fact describes the data on average.

[9] Engels J., Mashkevich S., Scheideler T., and Zinovjev G. Critical behavior of $\mathrm{SU}(2)$ lattice gauge theory. A complete analysis with the $\chi^{2}$-method. Phys. Lett. B, 365:219, 1996.

[10] Houdayer J. and Hartmann A. K. Low-temperature behavior of two-dimensional Gaussian Ising spin glasses. Phys. Rev. B, 70:014418, 2004. S measures the mean square distance of the scaled data to the master curve in units of standart errors.

[11] Schakel A. M. J. Percolation, Bose-Einstein condensation, and string proliferation. Phys. Rev. E, 63(2):026115, 2001.

[12] Ahuja R. K., Magnanti T. L., and Orlin J. B. Network Flows: Theory, Algorithms, and Applications. Prentice Hall, 1993.

[13] Hartmann A. K. and Rieger H. Optimization Algorithms in Physics. Wiley-VCH, Weinheim, 2001.

[14] Schwartz N., Nazaryev A., and Havlin S. Optimal paths in two and three dimensions. Phys. Rev. E, 58:7642, 1998.

[15] Melchert O. and Hartmann A. K. Fractal dimension of domain walls in two-dimensional Ising spin glasses. Phys. Rev. B, 76(17), 2007.

[16] Melchert O. and Hartmann A. K. Negative-weight percolation. New. J. Phys., 10:043039, 2008.

[17] Pfeiffer F. O. and Rieger H. Critical properties of loop percolation models with optimization constraints. Phys. Rev. E, 67(5):056113, 2003.

[18] Manna S. S. Structure of backbone perimeters of percolation clusters. Journal of Physics A: Mathematical and General, 22(4):433-440, 1989.

[19] Wenzel S., Bittner E., Janke W., Schakel A. M. J., and Schiller A. Kertész Line in the Three-Dimensional Compact U(1) Lattice Higgs Model. Phys. Rev. Lett., 95(5):051601, 2005.

[20] Cook W. and Rohe A. Computing minimum-weight perfect matchings. INFORMS J. Computing, 11:138-148, 1999. 